\title{
FUZZY SLIDING-MODE TEMPERATURE-CONTROL SYSTEM FOR SOAKING AND GERMINATION OF RICE SEEDS
}

\author{
Zheng Zhou ${ }^{1}$, Chunying Liang ${ }^{{ }^{*}}$ \\ ${ }^{1 *}$ Corresponding author. Heilongjiang Bayi Agricultural University/ Daqing, China. \\ E-mail: ndliangchunying@163 | ORCID ID: https://orcid.org/0000-0002-4957-2451
}

\section{KEYWORDS}

fuzzy sliding-mode control, temperature controller, soaking; germination.

\begin{abstract}
The soaking and germination process of rice seeds is the starting point in rice cultivation in cold regions and has a significant effect on grain yield. Efficient techniques for controlling the water temperature in the seed tank are required to enhance germination quality. This paper introduces a fuzzy theory for designing a fuzzy logic-based sliding mode controller (SMC) system for a rice seed soaking and germination device. The proposed system was theoretically and experimentally investigated to determine the efficiency of the soaking temperature-control system. A modified fuzzy SMC based on exponent approaching law is also presented for optimizing the proposed controller. A proportional integral derivative (PID) controller was designed to identify and compare the advantages of the proposed controllers. A comparative study of the computer simulation demonstrates that the performance of fuzzy SMC and modified fuzzy SMC are acceptable, and that both SMCs are superior to the PID controller. Furthermore, compared with the fuzzy SMC system, a reduction in electric energy consumption was observed for the modified fuzzy SMC. Moreover, both SMCs yielded similar soaking qualities.
\end{abstract}

\section{INTRODUCTION}

Rice is one of the most consumed cereals and is a staple food for more than half of the world's population (Saman et al., 2008). Nearly two-thirds of people worldwide depend on rice for at least $20 \%$ of their daily calories (Khamsen et al., 2016). Rice is widely cultivated in northeast China (Ye et al., 2018). Seed presoaking process is required because the growth cycle of rice in cold areas is shorter with a lower annual accumulated temperature (Deng et al., 2015). As the starting point of rice production, the germination rate in the seed presoaking process has a significant effect on the grain yield and depends primarily on the temperature of the water in the seed soaking tank. Deterioration of the biological quality and nutritional value, or even widespread death of rice seeds, commonly occurs owing to high water temperature during the soaking process; a low germination rate is usually observed if the soaking temperature dose not reach the required standard. Both cases result in the reduction of rice yield and quality. Therefore, precise control over the water temperature is imperative during the rice seed soaking and germination process.

Previous studies have been carried out on different temperature control systems in various applications. PID or PID modified controllers are widely used because of their simplicity and practical application (Shi et al., 2012; Zhang et al., 2011; Esfahani et al., 2015; Skjong \& Pedersen, 2016). A fuzzy PID control method has been presented to achieve the real-time temperature control of radiofrequency ablation (Cheng et al., 2017). A particle swarm optimization-based PID controller was designed for an air heater temperature-control system (Sungthong \& Assawinchaichote, 2016). A heat pump dryer based on a PID controller for fruit drying was designed by Ceylan et al. (2007). However, PID controllers cannot satisfy the accuracy requirements for certain industry cases, prompting the development of different control techniques. Both sliding-mode controllers (SMCs) and modified SMCs are commonly used control schemes owing to their stability and robustness (Tayebihaghighi et al., 2018). A sliding mode controller was

\footnotetext{
${ }^{1}$ Heilongjiang Bayi Agricultural University/ Daqing, China.

Area Editor: Jefferson Vieira José

Received in: 6-23-2019

Accepted in: 2-19-2020
} 
utilized for an energy-saving automotive air-conditioning system (Huang et al., 2017). Two sliding mode controllers were developed for a tempered glass furnace system to regulate the glass plate temperature (Almutairi \& Zribi, 2016). Traditional seed soaking and germination equipment is generally used for small-scale rice production, but an intelligent control system is required due to the increase in the rice production scale in northeastern China. For rice germination temperature-control systems, studies on controller design are rarely reported in the literature, although this system is extensively required in cold regions where rice is cultivated.

This paper presents a fuzzy logic-based SMC method for a rice-soaking device to address this problem, and discusses a modified SMC strategy. A fuzzy sliding controller was used to convert the tracking error to sliding-mode function, making the sliding mode function $s$ equal to zero. A Hammerstein-Wiener model was used to design the soaking and germination processes of the rice seeds. The simulation results obtained using the proposed fuzzy SMC and an optimized method with different parameter values were analyzed. A comparison between the PID controller results and the simulation results was performed to demonstrate the superiority of the fuzzy SMC. Furthermore, the experimental results revealed the control performance and germination quality achieved using the fuzzy SMC and modified fuzzy SMC.
The reminder of this paper is organized as follows: a rice-soaking model was built, and fuzzy control sliding mode controller and optimized fuzzy control sliding mode controller were designed, which are presented in Section 2. Simulation and experimental results are presented, and an analysis of the two proposed control algorithms and the PID method are carried out in Section 3. Finally, the conclusions derived from the study are stated in Section 4.

\section{MATERIAL AND METHODS}

\section{Model description of the rice seed soaking and germination system}

\section{Rice seed soaking and germination device}

The schematic diagram of the rice seed soaking and germination device is shown in Figure 1. The device was composed of a tank with a capacity of $9 \mathrm{~m}^{3}$, pump, electrical heater, water injection valve, drain valve, spray valve, sprayer, back-pressure valve, and water pipes. A heater with a maximum power of $2 \mathrm{~kW}$ was installed at the bottom of the tank. The water injection and drain valves were applied to exchange water from outside. In the seed tank, water was pumped to the heater pipe and then heated and injected into the tank through the sprayer, which circulated water. The back-pressure valve was employed to prevent the water from flowing backward.

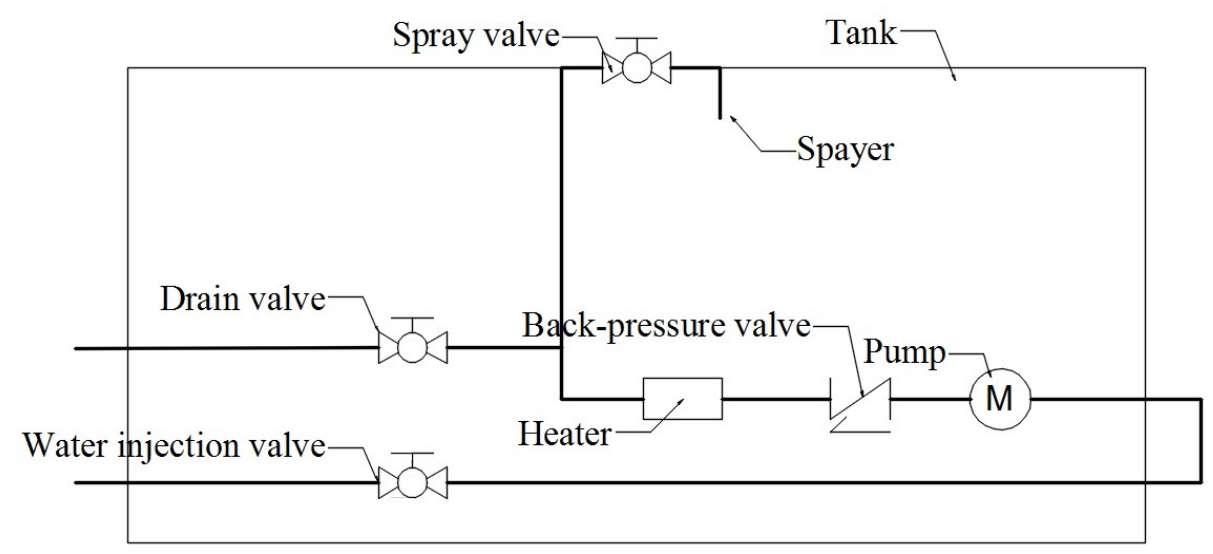

FIGURE 1. Schematic diagram of the rice seed soaking and germination device.

\section{Modeling of the system}

Theoretical models are usually used to analyze the physical and physiological properties of industrial process involving large numbers of parameters and high orders, which could complicate the implementation of real-time controller design. Therefore, previous studies typically involved system simplification and parameter reduction in control systems. In a generator excitation system, a mathematical model has been simplified using a novel sensitivity analysis method (Moghaddam et al., 2015). In a temperature control system, a linear model based on an input methodology of the neuro-fuzzy auto regressive external model has been built to design the controller (Ashkezari et al., 2017). In this study, the opening degree of the heat valve and sprayer are considered as inputs, while the water temperature in the tank is considered as the output. A Hammerstein-Wiener model is utilized for describing the rice seed soaking and germination system, which is expressed as follows:

$$
\begin{aligned}
& y(t)=G(w(t)) \\
& A\left(q^{-1}\right) \cdot w(t)=B\left(q^{-1}\right) \cdot v(t) \\
& v(t)=F(u(t))
\end{aligned}
$$


Where:

$u(t)$ and $y(t)$ are the input and output of the system, respectively;

$v(t)$ and $w(t)$ are the intermediate variables that define the input and output of the linear block, respectively;

$F(u)$ and $G(w)$ are nonlinear functions;

$q^{-1}$ is the backward shift operator; and

$A\left(q^{-1}\right)$ and $B\left(q^{-1}\right)$ denote the denominator and numerator of the transfer function of its dynamical part, respectively.

$A\left(q^{-1}\right), B\left(q^{-1}\right), F(u)$, and $G(w)$ are defined in eqs (4)-(7):

$$
\begin{aligned}
& A\left(q^{-1}\right)=1+\sum_{i=1}^{n_{A}} a_{i} q^{-i} \\
& B\left(q^{-1}\right)=q^{-d} \sum_{j=1}^{n_{B}} b_{j-d} q^{-j+d}
\end{aligned}
$$

$$
\begin{aligned}
& F(u)=\sum_{m=1}^{m_{F}} f_{m} u^{m} \\
& G(w)=\sum_{n=1}^{n_{C}} g_{n} w^{n}
\end{aligned}
$$

Where:

$d$ represents the time delay,

$n_{A}, n_{B}, m_{F}$, and $\mathrm{n}_{\mathrm{C}}$ are the degrees of $A\left(q^{-1}\right), B\left(q^{-1}\right)$, $F(u)$, and $G(w)$, respectively.

The Hammerstein-Wiener model was linearized to simplify the nonlinear system and is given in [eq. (8)].

$$
x(k+1)=A x(k)+B u(k)
$$

The frequency response curves of the Hammerstein-Wiener model and linearized model are shown in Figure 2, which demonstrated the validity of the linearized model.
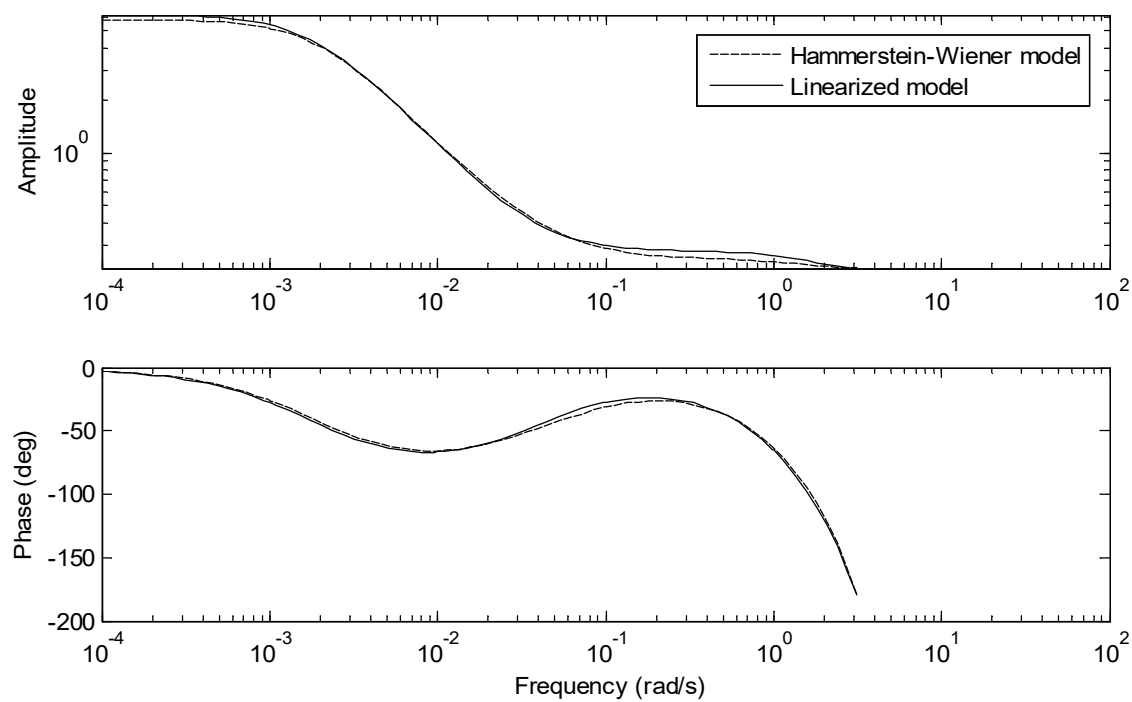

FIGURE 2. Frequency responses of the Hammerstein-Wiener model and linearized model.

\section{Controller design}

\section{Fuzzy SMC}

The functions $r(k)$ and $y(k)$ represent the input and output of the system, respectively; $e(k)$ is the error; and de(k) is the change rate of $e(k)$. The variables $e(k)$ and $\mathrm{d} e(k)$ are calculated as shown in [eq. (9)]:

$$
e(k)=r(k)-y(k), \mathrm{d} e(k)=\mathrm{d} \frac{e(k)-e(k-1)}{T}
$$

The switching function of SMC is expressed as follows:

$$
s(k)=c e(k)+d e(k)=C X(k)
$$

Where:

$C=[c, 1]$ is the switching function determined by the value of the sliding surface parameter $c$. The variation rate of $s(k)$ is expressed as follows:

$$
\mathrm{d} s(k)=s(k)-s(k-1)
$$

Where:

$s$ and $\dot{s}$ are the fuzzy variable quantities of $s(k)$ and $\mathrm{d} s(k)$, respectively, which are also the inputs of the fuzzy controller. The controlled variable $u$ is dependent on the fuzzy controller, and its variable quantity is $\Delta u . \Delta U$ is the fuzzy variable quantity, which is the output of the fuzzy controller. The fuzzy language set of $s, \dot{s}$, and $\Delta U$ is $\{P B, P N, P S, N S$, 
$N M, N B\}$, where $P B$ is "positive big," $P M$ is "positive middle," $P A$ is "positive small," $N S$ is "negative small," $N M$ is "negative middle," and $N B$ is "negative big." The fuzzy set universes of $s, \dot{s}$, and $\Delta U$ are given in eqs (12)-(14), the fuzzy control rules are presented in Table 1, and the membership functions of $s, \dot{s}$, and $\Delta U$ are shown in Figure 3.

$$
\begin{aligned}
& s=\{-3,-2,-1,0,+1,+2,+3\} \\
& \dot{s}=\{-3,-2,-1,0,+1,+2,+3\} \\
& \Delta U=\{-3,-2,-1,0,+1,+2,+3\}
\end{aligned}
$$

TABLE 1. Fuzzy control rules.

\begin{tabular}{ccccccccc}
\hline s & $\dot{s}$ & NB & NM & NS & ZO & PS & PM & PB \\
\hline & PB & ZO & PS & PM & PB & PB & PB & PB \\
& PM & NS & ZO & PS & PM & PB & PB & PB \\
PS & NM & NS & ZO & PS & PM & PB & PB \\
ZO & NB & NM & NS & ZO & PS & PM & PB \\
NS & NB & NB & NM & NS & ZO & PS & PM \\
NM & NB & NB & NB & NM & NS & ZO & PS \\
NB & NB & NB & NB & NB & NM & NS & ZO \\
\hline
\end{tabular}

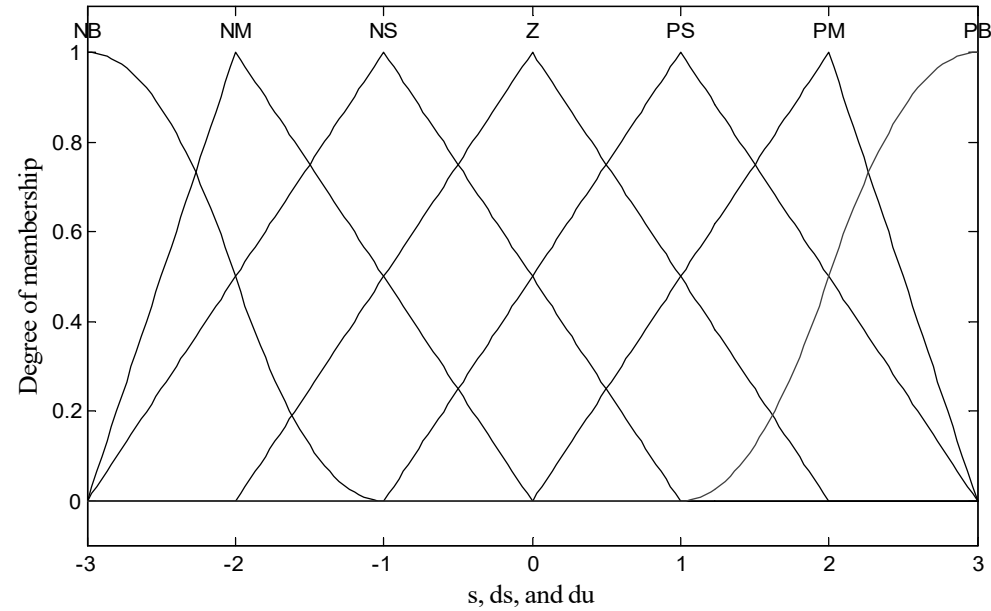

FIGURE 3. Membership functions of $s, \dot{s}$, and $\mathrm{d} u$ for fuzzy SMC.

\section{Modified fuzzy SMC}

An exponential law is adopted to prevent the large flux and torque ripple of the fuzzy SMC. The function $r(k)$ is the position instructor and $\mathrm{d} r(k)$ is its derivative. Based on the linear extrapolation method, $r(k+1)$ and $\mathrm{d} r(k+1)$ yield to the expression in [eq. (15)].

$r(k+1)=2 r(k)-r(k-1), \mathrm{d} r(k+1)=2 \mathrm{~d} r(k)-\mathrm{d} r(k-1)$

The switching function is given as follows:

$$
s(k)=C_{e} E=C_{e}(R(k)-x(k))
$$

Where:

$C_{e}=[c, 1]$. The switching function $C e$, which affects the stability, and the response time are determined by the value of the sliding surface parameter $c$.
The control law based on the exponential reaching method is represented as follows:

$$
\begin{gathered}
u(k)=(C e B)-1(C e R(k+1)-C e A x(k)-s(k)-\mathrm{d} s(k)) \\
\mathrm{d} s(k)=\varepsilon T \operatorname{sgn}(s(k))-q T s(k)
\end{gathered}
$$

Where:

$\varepsilon$ is the absolute value of the fuzzy output $f s(k)$, which has a significant effect on system chattering. $q$ is a parameter in the SMC based on an exponent approach law, which is the significant factor that influences the dynamic transition. The membership functions of $s, \dot{s}$, and $\varepsilon$ are shown in Figure 4. 


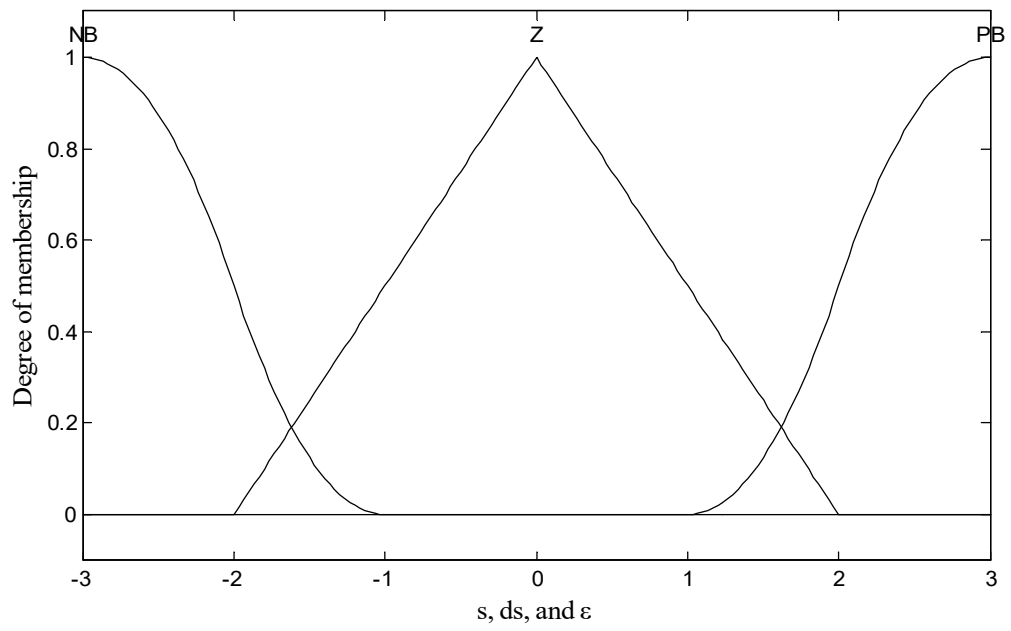

FIGURE 4. Membership functions of $s, \dot{s}$, and $\varepsilon$ for modified fuzzy SMC.

\section{RESULTS AND DISCUSSION}

\section{Simulated control results and discussion}

In this section, the proposed control strategy simulation is carried out using MATLAB ${ }^{\circledR}$ software, and the simulation results with different control parameters are analyzed. Because PID controllers are widely used in temperature control for industrial applications (Monje et al., 2008; Bennett, 2001), a PID simulation result is also presented to compare the simulation results with the results of the proposed fuzzy SMC and modified SMC. Temperature guided by the technological schedule at each stage of the rice seed soaking and germination process were different. Hence, the reference temperature used in the simulation was a step function. The reference signal was set at $20{ }^{\circ} \mathrm{C}$, the sampling period was $1 \mathrm{~s}$, and the total simulation time was $1000 \mathrm{~s}$.

Figure 5 depicts the performance comparison among the fuzzy SMCs with $c=1, c=100$, and $c=200$. All the three step response curves could raise the setpoint directly without overshooting and could maintain the temperature at that level. The rise-time is shortest for $c=1$ and longest for $c=200$. For the fuzzy SMC, the rise-time increases with $c$.

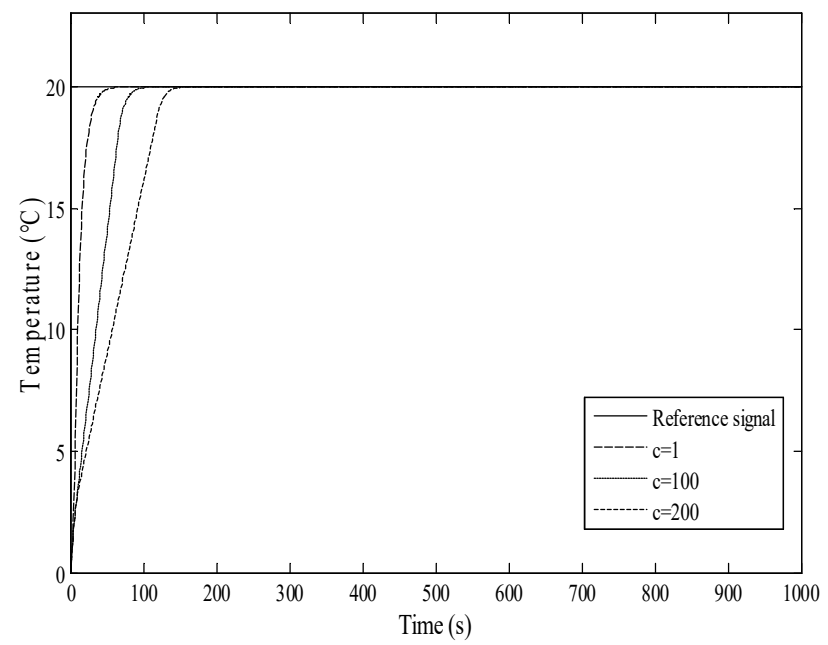

FIGURE 5. Performance comparison of the fuzzy SMC.

Figure 6 displays the performance of the modified fuzzy SMC with $c=0.1, c=2$, and $c=4$. The temperature with $c=0.1, c=2$, and $c=4$ reaches the setpoint in 134, 74, and $65 \mathrm{~s}$, respectively, and the overshoot values are 18.4\%, $11.07 \%$, and $10.00 \%$, respectively. The rise-time is shortest for $c=4$ and the longest for $c=0.1$, while the overshoot is the smallest for $c=4$ and the largest for $c=0.1$. Hence, the modified fuzzy SMC for $c=4$ exhibits the best performance owing to its smallest overshoot and shortest rise-time. 


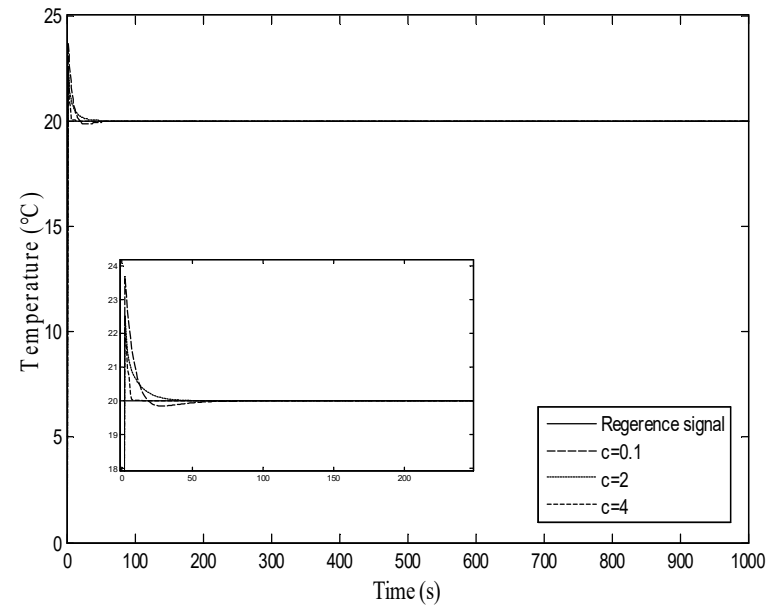

FIGURE 6. Performance comparison of the modified fuzzy SMC.

The performance comparison for the PID controller, fuzzy SMC, and modified fuzzy SMC is given in Figure 7. The control parameter $c$ used for the fuzzy SMC and modified fuzzy SMC are equal to 2 and 4 , respectively. The gains in the PID controller are as follows: $k_{p}=0.001, k_{i}=$ 0.00001 , and $k_{d}=0.01$. The performance of the PID controller was inferior to the other two controllers, which was in sharp contrast to the overshoot and settling time of the fuzzy SMC and modified fuzzy SMC. For the two proposed control algorithms, the modified fuzzy SMC significantly improves its performance compared with the fuzzy SMC for rise-time. The output of the modified fuzzy SMC increases rapidly in an almost straight-line manner towards the setpoint, then suddenly changes its direction immediately the setpoint is reached, and then becomes stable at the setpoint. However, the improvement in rise-time occurs at the expense of the overshoot, which deteriorates for the modified fuzzy SMC. Table 2 lists the performance indices of the three controllers.

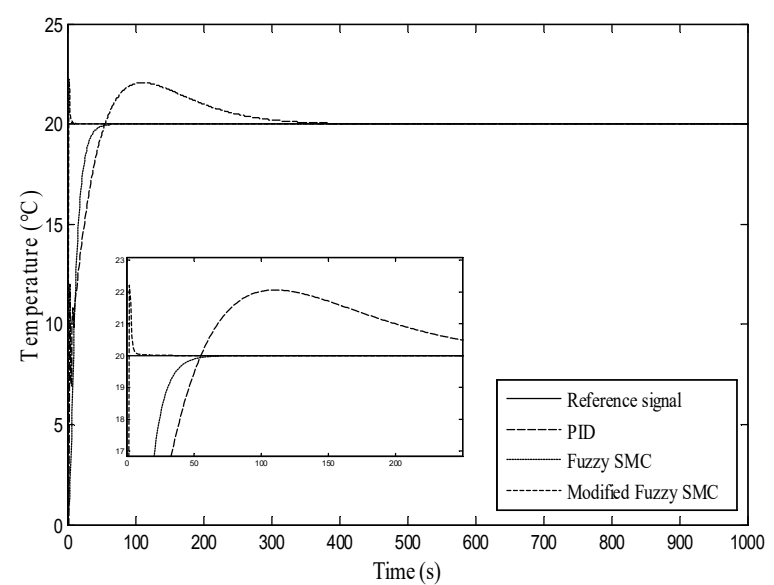

FIGURE 7. Performance comparison of the PID controller, fuzzy SMC, and modified fuzzy SMC.

TABLE 2. Control performance indices of the PID controller, fuzzy SMC, and modified fuzzy SMC.

\begin{tabular}{lccc}
\hline & Rise-time (s) & Settling-time (s) & Maximum overshoot (\%) \\
\hline PID controller & 111 & 432 & 10.32 \\
Fuzzy SMC & - & 119 & - \\
Modified fuzzy SMC & 2 & 65 & 10.00 \\
\hline
\end{tabular}

\section{Experimental results and discussion}

The rice seeds used for the experimental tests were of Kongyu 131 variety and had the same quality; these seeds were extensively planted in Heilongjiang Province, China. A small-sized rice seed soaking and germination device was developed in Heilongjiang Bayi Agricultural University to evaluate the control performance of the proposed scheme, and a photograph of the actual device is shown in Figure 8. The soaking tank had a size of $3 \mathrm{~m} \times 2 \mathrm{~m} \times 1.5 \mathrm{~m}$. The water injection and drain valves were connected to an outside water supply and drainage system. The temperature of the heater and the opening degree of the sprayer, as the input variables, were continuously changeable, and the output was 
the average value measured using the temperature sensors separately installed in a different layer of the tank. Water for soaking and germination of the seeds was collected from a well, and the initial temperature of the water in the tank was $3.5{ }^{\circ} \mathrm{C}$. These conditions stated above were selected to simulate the actual conditions of the soaking and germination of rice seeds, as used in the industry. The seed soaking and germination temperature control results obtained using the fuzzy sliding SMC and modified fuzzy SMC are presented in Figure 9. The entire soaking process required $686 \mathrm{~h}$, and both the controllers achieved a satisfactory temperature performance. Improvements were observed in the settling time reduction of the modified fuzzy SMC. However, the fluctuation in the response curve was larger than fuzzy SMC when the temperature was adjusted at each stage, which matched the response curves in the simulation. Table 3 lists the performance indices of the experimental results. In terms of energy consumption, the modified fuzzy SMC exhibited an enhanced performance owing to its shorter settling time: its energy consumption amounted to $6729 \mathrm{~kW} \cdot \mathrm{h}$, which is $165 \mathrm{~kW} \cdot \mathrm{h}$ less than the energy consumed by the fuzzy SMC.

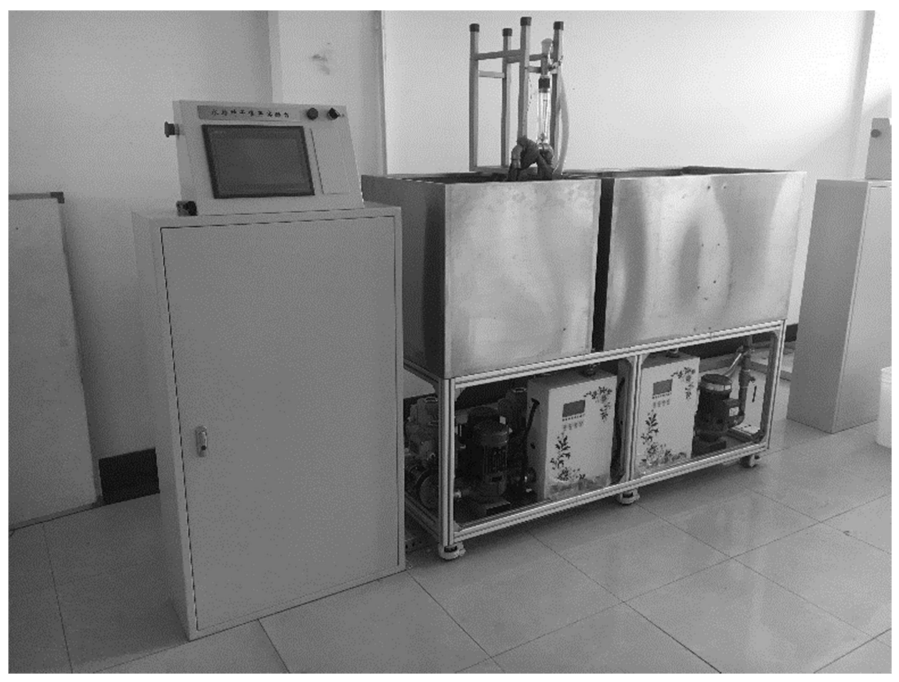

FIGURE 8 Rice seed soaking and germination device.

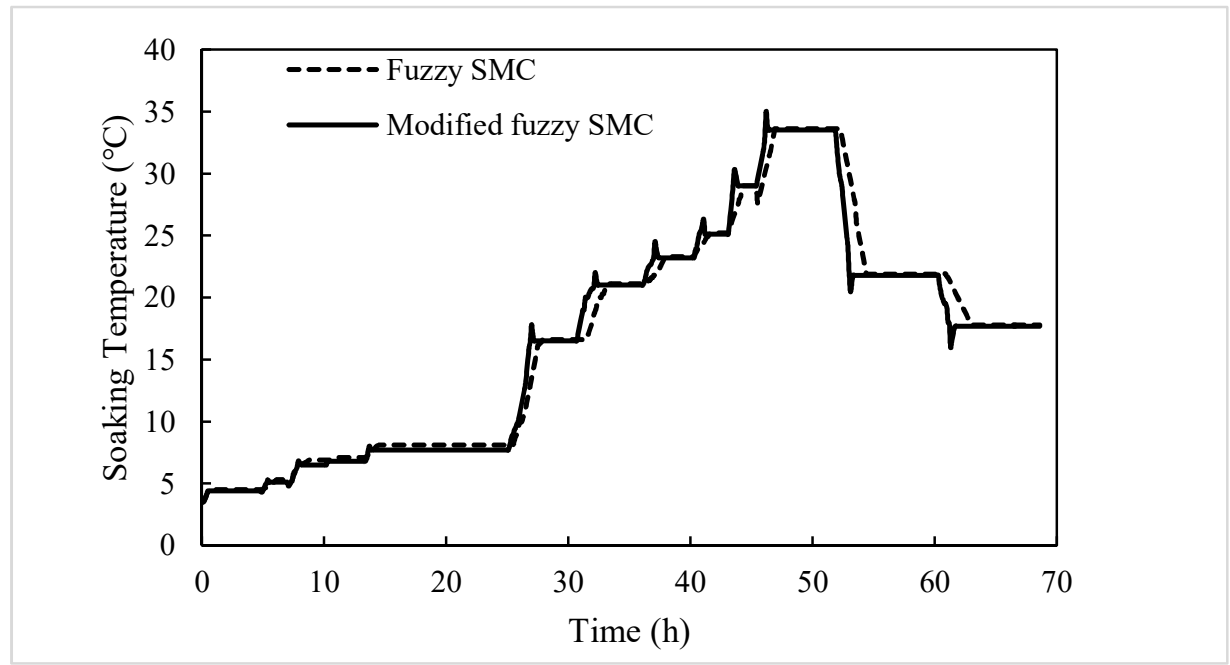

FIGURE 9. Soaking temperature of fuzzy SMC and modified fuzzy SMC.

TABLE 3. Indices of the experimental results.

\begin{tabular}{lcccc}
\hline & Sprout rate & Death rate & Germination potential & Electricity consumption $(\mathrm{kW} \cdot \mathrm{h})$ \\
\hline Fuzzy SMC & $93 \%$ & $3 \%$ & $90 \%$ & 6894 \\
Modified fuzzy SMC & $94 \%$ & $4 \%$ & $90 \%$ & 6729 \\
\hline
\end{tabular}




\section{CONCLUSIONS}

In this study, a fuzzy SMC and modified fuzzy SMC were developed for a rice soaking and germination device. The application of the two proposed controllers demonstrated their effectiveness through the simulation and experimental test. The performance of the two controllers with different parameter values were examined, and the dynamic and steady-state performance of the proposed control schemes were found to be acceptable. The control behavior of the modified fuzzy SMC was superior to that of the fuzzy SMC, which led to a shorter rise-time and faster convergence. From the experimental tests, the germination quality via the two techniques both gave excellent results. In terms of energy consumption, the modified fuzzy SMC was more energy-saving.

\section{ACKNOWLEDGEMENTS}

This study was financially supported by University Nursing Program for Young Scholars with Creative Talents in Heilongjiang Province (NO. UNPYSCT-2018083) and Heilongjiang Farms and Land Reclamation Administration Key Point Research Project (No. HKKYZD190806).

\section{REFERENCES}

Almutairi NB, Zribi M (2016) Sliding mode controllers for a tempered glass furnace. ISA Transactions 60:21-37.

Ashkezari FD, Piltan F, Sarostad M, Sulaiman NB (2017) Design Nonlinear Model-free Neuro-fuzzy ARX Algorithm to Control of System's Temperature. International Journal of Smart Home 11(1):117-130.

Bennett S (2001) The past of PID controllers. Annual Reviews in Control 25:43-53.

Ceylan İ, Aktas M, Dogan H (2007) Mathematical modeling of drying characteristics of tropical fruits, Applied Thermal Engineering 27(11-12):1931-1936.

Cheng Y, Nan Q, Wang R, Dong T, Tian Z (2017) Fuzzy proportional integral derivative control of a radiofrequency ablation temperature control system. In: 10th International Congress on Image and Signal Processing, Biomedical Engineering and Informatics. Shanghai, Proceedings...

Deng N, Ling X, Sun Y, Zhang C, Fahad S, Peng S, Cui K, Nie L, Huang J (2015) Influence of temperature and solar radiation on grain yield and quality in irrigated rice system. European Journal of Agronomy 64:37-46.

Esfahani N, Azimirad V, Danesh M (2015) A Time Delay Controller included terminal sliding mode and fuzzy gain tuning for Underwater Vehicle-Manipulator Systems. Ocean Engineering 107:97-107.
Fu L, Tola E, Al-Mallahi A, Li R, Cui Y (2019) A novel image processing algorithm to separate linearly clustered kiwifuits. Biosystems Engineering 183:184-195.

Huang Y, Khajepour A, Ding H, Bagheri, F, Bahrami M (2017) An energy-saving set-point optimizer with a sliding mode controller for automotive air-conditioning/refrigeration systems. Applied Energy 188:576-585.

Khamsen N, Onwimol D, Teerakawanich N, Dechanupaprittha S, Kanokbannakorn W, Hongesombut K, Srisonphan S (2016) Rice (Oryza sativa L.) seed sterilization and germination enhancement via atmospheric hybrid nothermal discharge plasma. ACS Applied Materials and Interfaces 8(30):19268-19275.

Moghaddam IN, Salami Z, Easter L (2015) Sensitivity analysis of an excitation system in order to simplify and validate dynamic model utilizing plant test data. IEEE Transactions on Industry Application 51(4):3435-3441.

Monje CA, Vinagre BM, Feliu V, Vhen YQ (2008). Tuning and auto-tuning of fractional order controllers for industry applications. Control Engineering Practice 16(7):798-812.

Saman P, Vazquez JA, Pandiella SS (2008) Controlled germination to enhance the functional properties of rice. Process Biochemistry 43(12):1377-1382.

Shi D, Gao G, Gao Z, Xiao P (2012) Application of Expert Fuzzy PID Method for Temperature Control of Heating Furnace. Procedia Engineering 29:257-261.

Skjong S, Pedersen E (2016) Model-based control designs for offshore hydraulic winch systems. Ocean Engineering 121:224-238.

Sungthong A, Assawinchaichote W (2016) Particle Swam Optimization Based Optimal PID Parameters for Air Heater Temperature Control System. Procedia Computer Science 86:108-111.

Tayebihaghighi S, Piltan F, Kim J (2018) Control of an Uncertain Robot Manipulator Using an Observation-based Modified Fuzzy Sliding Mode Controller. International Journal of Intelligent Systems and Applications 10(3):41-49.

Ye J, Niu X, Yang Y, Wang S, Xu Q, Yan X, Yu H, Wang Y, Wang S, Feng Y, Wei X (2018) Divergent Hd1, Ghd7, and DTH7 Alleles Control Heading Date and Yield Potential of Japonica Rice in Northeast China. Front Plant Science $9: 35$.

Zhang H, Shi Y, Mehr A (2011) Robust static output feedback control and remote PID design for networked motor system. IEEE Transactions on Industry Electronics 58(12):5396-5405. 